\title{
Identifying sources of non-stationary neural ensemble dynamics
}

\author{
Emili Balaguer-Ballester ${ }^{1,2^{*}}$, Hamid Bouchachia', Christopher C Lapish ${ }^{3}$ \\ From Twenty Second Annual Computational Neuroscience Meeting: CNS*2013 \\ Paris, France. 13-18 July 2013
}

In the traditional view on brain activity dynamics, the cognitive flow of information wanders through multiple stable states driven by task-dependent inputs [1-3]. This focus has been recently challenged both empirically and from the modeling perspective. For instance, experimental studies suggest that olfactory [4] and gustatory representations [5] can be understood as a sequence of temporally stable, attractor-like states; but such transient states are essentially transient and driven by stochastic fluctuations. Likewise, in several contemporary models, intrinsic activity fluctuations can drive default transitions between states $[6,7]$.

It has been recently proposed that such transient states are basically shaped by anatomical connectivity and transitions between them occur even in the absence of external stimuli [8]: Noise enriches the dynamical repertoire of deterministic states; creating flexible 'ghost' attractors which enable the effective processing of taskrelated cognitive entities [7].

A different metaphor of transient brain dynamics was proposed by Rabinovich and colleagues [9]. In such model, transitions between states mapping cognitive entities is purely deterministic: The dynamical portrait of the model consists of successions of temporally stable states i.e. metastable saddle points linked by heteroclinic channels. Such dynamical objects are particularly reliable, but neural activity eventually switches between them even without the intervention of noise or external inputs.

In this work we develop an empirical criterion to discern whether observable neural ensemble activity can be originated by non-autonomous yet deterministic dynamical systems or rather by stochastic fluctuations between temporally attracting states. Towards this goal, we used in

\footnotetext{
* Correspondence: eb-ballester@bournemouth.ac.uk

'School of Engineering and Computing, Bournemouth University, Bournemouth, BH12 5BB, UK

Full list of author information is available at the end of the article
}

vivo multiple single-unit recording in rodent frontal cortex during a decision making task. Effective dynamics of neural activity is first empirically reconstructed in nonlinear state spaces [10]. Then, trajectory analyses enable us to differentiate systems driven by non-automatous dynamics from those driven by stochastic transitions.

\section{Conclusions}

Underlying dynamics of recorded ensemble activity is probably driven by a slowly drifting, non-autonomous dynamical system containing low-order nonlinear interactions.

\section{Author details}

${ }^{1}$ School of Engineering and Computing, Bournemouth University, Bournemouth, BH12 5BB, UK. ${ }^{2}$ Bernstein Center for Computational Neuroscience, Zl Mannheim-University of Heidelberg, Mannheim, J5, Germany. ${ }^{3}$ Department of Psychology, Indiana University-Purdue University, Indianapolis, Indiana, 46202, USA.

Published: 8 July 2013

\section{References}

1. Hopfield JJ: Neural networks and physical systems with emergent collective computational abilities. Proc Natl Acad Sci USA 1982, 79:2554-2258.

2. Wilson HR: Spikes, decisions, and actions: The dynamical foundations of neuroscience. New York: Oxford University Press; 1999.

3. Wills TJ, Lever C, Cacucci F, Burgess N, O'Keefe J: Attractor dynamics in the hippocampal representation of the local environment. Science 2005, 308:873-876.

4. Niessing J, Friedrich RW: Olfactory pattern classification by discrete neuronal network states. Nature 2010, 465:47-54.

5. Miller P, Katz DB: Stochastic transitions between neural states in taste processing and decision-making. J Neurosci 2010, 30:2559-2570.

6. Maass W, Natschläger T, Markram H: Real-time computing without stable states: a new framework for neural computation based on perturbations. Neural Comput 2002, 14:2531-2560.

7. Deco G, Jirsa V: Ongoing cortical activity at rest: criticality, multistability and ghost attractors. J Neurosci 2012, 32:3366-3375.

8. Deco G, Jirsa V, Mclntosh AR: Emerging concepts for the dynamical organization of resting- state activity in the brain. NatRev Neurosci 2011, 12:43-56.
C Biomed Central

다 2013 Balaguer-Ballester et al; licensee BioMed Central Ltd. This is an Open Access article distributed under the terms of the Creative Commons Attribution License (http://creativecommons.org/licenses/by/2.0), which permits unrestricted use, distribution, and reproduction in any medium, provided the original work is properly cited. 
9. Rabinovich M, Huerta R, Laurent G: Transient dynamics for neural processing. Science 2008, 321:48-50

10. Balaguer-Balllester E, Lapish C, Seamans JK, Durstewitz D: Attracting Dynamics of Frontal Cortex Ensembles during Memory-Guided DecisionMaking. PLoS Comput Biol 2011, 7(5):e100205, May.

\section{doi:10.1186/1471-2202-14-S1-P15}

Cite this article as: Balaguer-Ballester et al: Identifying sources of nonstationary neural ensemble dynamics. BMC Neuroscience 2013 14(Suppl 1):P15.

Submit your next manuscript to BioMed Central and take full advantage of:

- Convenient online submission

- Thorough peer review

- No space constraints or color figure charges

- Immediate publication on acceptance

- Inclusion in PubMed, CAS, Scopus and Google Scholar

- Research which is freely available for redistribution 